\title{
Frequency ratios and the perception of tone patterns
}

\author{
E. GLENN SCHELLENBERG \\ University of Windsor, Windsor, Ontario, Canada \\ and \\ SANDRA E. TREHUB \\ University of Toronto, Mississauga, Ontario, Canada
}

\begin{abstract}
We quantified the relative simplicity of frequency ratios and reanalyzed data from several studies on the perception of simultaneous and sequential tones. Simplicity of frequency ratios accounted for judgments of consonance and dissonance and for judgments of similarity across a wide range of tasks and listeners. It also accounted for the relative ease of discriminating tone patterns by musically experienced and inexperienced listeners. These findings confirm the generality of previous suggestions of perceptual processing advantages for pairs of tones related by simple frequency ratios.
\end{abstract}

Since the time of Pythagoras, the relative simplicity of the frequency relations between tones has been considered fundamental to consonance (pleasantness) and dissonance (unpleasantness) in music. Most naturally occurring tones (e.g., the sounds of speech or music) are complex, consisting of multiple pure-tone (sine wave) components. Terhardt $(1974,1978,1984)$ has suggested that relations between different tones may be influenced by relations between components of a single complex tone. For single complex tones, including those of speech and music, the frequency values (in hertz, or cycles per second) of individual pure-tone components (called harmonics) are usually integer multiples of the fundamental frequency, or first (lowest) harmonic. For example, a complex tone with a fundamental frequency of $100 \mathrm{~Hz}$ consists of harmonics of $100,200,300,400,500 \mathrm{~Hz}$, and so on. Thus, relations between the lower and most readily perceived harmonics (Plomp, 1964) can be represented by small-integer frequency ratios such as $2: 1$ (between 200 and $100 \mathrm{~Hz}$ ), 3:2, 4:3, 5:3, 5:4, and so on.

Historically, intervals (i.e., combinations of two tones) formed by complex tones whose fundamental frequencies are related by simple (i.e., small-integer) ratios have been considered consonant, smooth, or pleasant; intervals with complex (i.e., large-integer) ratios have been considered dissonant, or unpleasant. Rameau (1722/1971) considered the consonance of intervals with simple frequency ratios to be a consequence of the simple ratios found among har-

This research was supported by grants from the Natural Sciences and Engineering Research Council of Canada. We thank Laurel J. Trainor for providing her data for reanalysis and Robert Crowder for his helpful comments on an earlier version of the manuscript. Send reprint requests to E. G. Schellenberg, Department of Psychology, University of Windsor, Windsor, Ontario, Canada N9B 3P4, or to S. E. Trehub, Centre for Research in Human Development, University of Toronto, Erindale Campus, Mississauga, Ontario, Canada L5L 1C6. monics of a single complex tone. Currently, the degree of perceived consonance is believed to result from both sensory and experiential factors. Whereas sensory consonance is constant across musical styles and cultures, musical consonance presumably results from learning what sounds pleasant in a particular musical style.

Helmholtz (1885/1954) proposed that the consonance of two simultaneous complex tones is a function of the ratio between their fundamental frequencies-the simpler the ratio, the more harmonics the tones have in common. For two complex tones that stand in a ratio of $2: 1$, half of the harmonics of the lower tone are present in the harmonic series of the higher tone, while all of the harmonics of the higher tone are present in the series of the lower tone. For tones that stand in a ratio of $3: 2$, one third of the harmonics of the lower tone are present in the series of the higher tone, while half of the harmonics of the higher tone are present in the series of the lower tone. Thus, amplitude fluctuations and sensations of beating arising from harmonics that are close but not identical in pitch are less likely between tones related by simple frequency ratios (more common harmonics) than between tones related by more complex ratios (fewer common harmonics).

Contemporary accounts of consonance and dissonance (Kameoka \& Kuriyagawa, 1969a, 1969b; Plomp \& Levelt, 1965) have incorporated the notion of critical bands into the earlier formulation (Helmholtz, 1885/1954). Critical bands are presumed to function like auditory filters. Each tone interacts with other tones within a certain range of surrounding frequencies (its critical band); beyond that range (i.e., when tones have nonoverlapping critical bands), such interactive effects are minimal. Thus, simultaneous pure tones that are proximate (but not identical) in pitch have overlapping critical bands, resulting in beating and the perception of dissonance, or 
roughness (Kameoka \& Kuriyagawa, 1969a; Plomp \& Levelt, 1965). The most dissonant interval occurs at about a quarter of a critical bandwidth (Plomp \& Levelt, 1965). The width of the critical band is a constant frequency ratio (between two and three semitones) for tones above $500 \mathrm{~Hz}$; under $500 \mathrm{~Hz}$, the critical band becomes wider (in number of semitones) as frequency decreases (Plomp $\&$ Levelt, 1965). Thus, intervals that are consonant in the mid- to upper ranges (e.g., intervals of three and four semitones) may be dissonant in the lower ranges; in musical compositions, these lower intervals are avoided (Huron \& Sellmer, 1992; Plomp \& Levelt, 1965). In sum, the consonance of simultaneous pure tones is considered a function of frequency distance (i.e., whether or not critical bands overlap) and absolute frequency, independent of the simplicity of frequency ratios.

For simultaneous complex tones, dissonance occurs when the critical bands of adjacent harmonics overlap but are not identical. Thus, complex tones related by simple frequency ratios are less likely to cause dissonance than those related by more complex ratios, not because of the simplicity of frequency ratios per se, but because the critical bands of adjacent harmonics are less likely to generate interference. In the case of successive tones, effects of ratio simplicity are thought to result primarily from exposure to a specific musical culture or style, illustrating the phenomenon of musical as opposed to sensory consonance. In the context of musical consonance, terms such as tonal affinity, compatibility, and stability are often used.

Because the degree of consonance/dissonance (sensory) is largely attributed to overlapping critical bands, the prevailing view is that the relation between frequency ratios and musical intervals is arbitrary, or coincidental (e.g., Dowling \& Harwood, 1986). Indeed, judgments of dissonance have been found to be independent of ratio simplicity (Plomp \& Levelt, 1965), as have judgments of similarity (Kallman, 1982). Moreover, the discrimination of changes in melodic (sequential) intervals by musically untrained listeners is also reported to be independent of the relative simplicity of frequency ratios (Burns \& Ward, 1978).

Trehub and her colleagues (Cohen, Thorpe, \& Trehub, 1987; Schellenberg, 1994; Schellenberg \& Trehub, in press; Trainor \& Trehub, 1993a, 1993b; Trehub, Thorpe, \& Trainor, 1990) contend, however, that tones related by simple frequency ratios are inherently easier to process than tones related by more complex ratios. They found processing advantages for simple frequency ratios in a task that required musically untrained adults and young children to detect changes in sequences of pure tones, where overlapping critical bands were not a factor (Schellenberg, 1994). They also found that discrimination accuracy improved with the increasing ratio simplicity of the patterns (Schellenberg \& Trehub, in press).

If simple frequency ratios have natural processing advantages (Schellenberg, 1994; Schellenberg \& Trehub, in press), they should predominate in musical scales crossculturally. Indeed, octaves, which exemplify the very sim- ple ratio of $2: 1$, are found in the music of virtually all cultures (Dowling \& Harwood, 1986; Lerdahl \& Jackendoff, 1983). Moreover, intervals with ratios of $3: 2$ (fifths) and 4:3 (fourths) have been identified in many cultures (Sachs, 1943), often functioning as stable intervals or points of resolution (Meyer, 1956).

In the present paper, we explore the perceptual consequences of ratio simplicity by examining interval-perception data from numerous investigators, representing a wide variety of listeners, listening contexts, and experimental tasks. Just as grammatical simplicity, defined linguistically, need not imply psychological simplicity, ratio simplicity, defined mathematically, need not have psychological consequences. The hypothesis under consideration is that simple frequency ratios confer perceptual processing advantages relative to complex ratios. Specifically, simple frequency ratios should facilitate the processing of patterns comprised of pure or complex tones in the context of melodic (sequential) or harmonic (simultaneous) intervals. Moreover, tones related by simple frequency ratios should be perceived as more consonant, or compatible, than tones related by more complex frequency ratios. To rule out exposure to Western music as the principal explanatory factor, these effects should be demonstrable with listeners from widely different musical cultures. The effects should be evident, moreover, relatively early in life. Finally, the hypothesized effects should be obligatory-apparent in musical as well as nonmusical contexts.

\section{QUANTIFYING THE SIMPLICITY OF FREQUENCY RATIOS}

Our index of ratio simplicity, essentially a modification of previous indexes (Levelt, van de Geer, \& Plomp, 1966; van de Geer, Levelt, \& Plomp, 1962), is the reciprocal of the natural logarithm of the sum of the two integers in a frequency ratio in its simplest form (i.e., no common factors in the integers). Thus, the simplicity value for ratio $\mathrm{X}: \mathrm{Y}$ is $\left[\log _{\mathrm{e}}(\mathrm{X}+\mathrm{Y})\right]^{-1}$. In the special case of simultaneous pure tones presented in phase, the index represents the inverse of the logarithm of the total number of complete cycles of sine waves in the period common to both tones. Table 1 presents all intervals from 0 to $12 \mathrm{semi-}$ tones that are multiples of semitones, their frequency ratios, and the calculation of the ratio-simplicity values. The frequency ratios are based on just tuning, a system in which notes are tuned to form small-integer ratios with the tonic of the scale (the tone called $d o$ ).

Burns and Ward's (1982, Table 1) presentation of frequency ratios for justly tuned intervals was the source of ratios for the index (intervals were limited to integer multiples of semitones). Frequency ratios of intervals between the tonic and other tones of the Western major scale are relatively consistent across authors (octaves as 2:1, fifths as 3:2, etc.), but those of other intervals tend to vary considerably (Burns \& Ward, 1982). For example, the tritone is sometimes considered to have a ratio of $64: 45$ in- 
Table 1

Interval Size (in Semitones) With Corresponding Interval Name, Justly Tuned Frequency Ratio, Sum of Integers in the Ratio, Logarithm of the Sum, and Inverse of the Logarithm

\begin{tabular}{clcccc}
\hline $\begin{array}{c}\text { Interval } \\
\text { Size }\end{array}$ & Interval & $\begin{array}{c}\text { Frequency } \\
\text { Ratio }\end{array}$ & $\begin{array}{c}\text { Sum of } \\
\text { Integers }\end{array}$ & $\begin{array}{c}\text { Logarithm } \\
\text { of Sum }\end{array}$ & $\begin{array}{c}\text { Reciprocal of } \\
\text { Logarithm }\end{array}$ \\
\hline 0 & unison & $1: 1$ & 2 & 0.693 & 1.443 \\
1 & minor second & $16: 15$ & 31 & 3.434 & 0.291 \\
2 & major second & $9: 8$ & 17 & 2.833 & 0.353 \\
3 & minor third & $6: 5$ & 11 & 2.398 & 0.417 \\
4 & major third & $5: 4$ & 9 & 2.197 & 0.455 \\
5 & perfect fourth & $4: 3$ & 7 & 1.946 & 0.514 \\
6 & tritone & $45: 32$ & 77 & 4.344 & 0.230 \\
7 & perfect fifth & $3: 2$ & 5 & 1.609 & 0.621 \\
8 & minor sixth & $8: 5$ & 13 & 2.565 & 0.390 \\
9 & major sixth & $5: 3$ & 8 & 2.079 & 0.481 \\
10 & minor seventh & $16: 9$ & 25 & 3.219 & 0.311 \\
11 & major seventh & $15: 8$ & 23 & 3.135 & 0.319 \\
12 & octave & $2: 1$ & 3 & 1.099 & 0.910 \\
\hline
\end{tabular}

Note-The reciprocal of the logarithm of the sum of integers is used as the index of simplicity of frequency ratios.

stead of the more conventional 45:32. For instances in which Burns and Ward (1982) provide two different ratios for a single interval, we selected the ratio that minimized deviation in size from its equal-tempered counterpart. (In the equal-tempered scale, an octave is divided into 12 equal steps, each an equal-tempered semitone. Thus, all equal-tempered intervals are integer multiples of equal-tempered semitones. Except for the octave, equaltempered intervals are slightly mistuned from exact simple ratios.) In the case of the tritone, where both ratios are equidistant from the equal-tempered interval, we selected the smaller integer ratio (45:32 rather than 64:45). Although these decision rules are arbitrary, the ratios on which the index is based are widely used for justly tuned intervals as well as for small-integer approximations to equal-tempered intervals, and they are identical to those reported by Krumhansl (1990) for intervals from 0 to 12 semitones.

The index is formulated from justly tuned intervals, but the resulting values are assumed to apply to intervals slightly mistuned from exact ratios, including equaltempered intervals. This assumption gains credence from the finding that performing musicians often produce small discrepancies in interval size (Rakowski, 1990), which listeners still perceive as being "in tune" (Burns \& Ward, 1982; Ward, 1970). Only one simplicity value is assigned to each interval, despite the possibility of justly tuned intervals with the same number of semitones having more than one ratio, depending on their position in the scale. The index can also be extended to intervals larger than 12 semitones (one octave) by doubling the first integer in the ratio of the interval 12 semitones smaller. For example, a major ninth (14 semitones) is an octave larger than a major second (2 semitones); its frequency ratio is $18: 8$, which can be reduced to $9: 4$, and its ratio-simplicity value is $\left[\log _{e}(9+4)\right]^{-1}=.391$.

As can be seen in Figure 1, which presents simplicity values for frequency ratios as a function of interval size and Western music-theoretic classifications of consonance/ dissonance, dissonant intervals have lower simplicity values than consonant intervals. Similarly, imperfect consonances have relatively lower values than perfect consonances. Intervals of 0 and 12 semitones (unison and octave, respectively) have the highest simplicity values (i.e., the simplest ratios), whereas the interval of 6 semitones (the tritone) has the lowest value (i.e., the most complex ratio). The simplicity values associated with these particular intervals are consistent with Narmour's (1992) suggestion that unisons, octaves, and tritones are inherently special, or "peculiar," with correspondingly distinctive perceptual properties. For example, Narmour

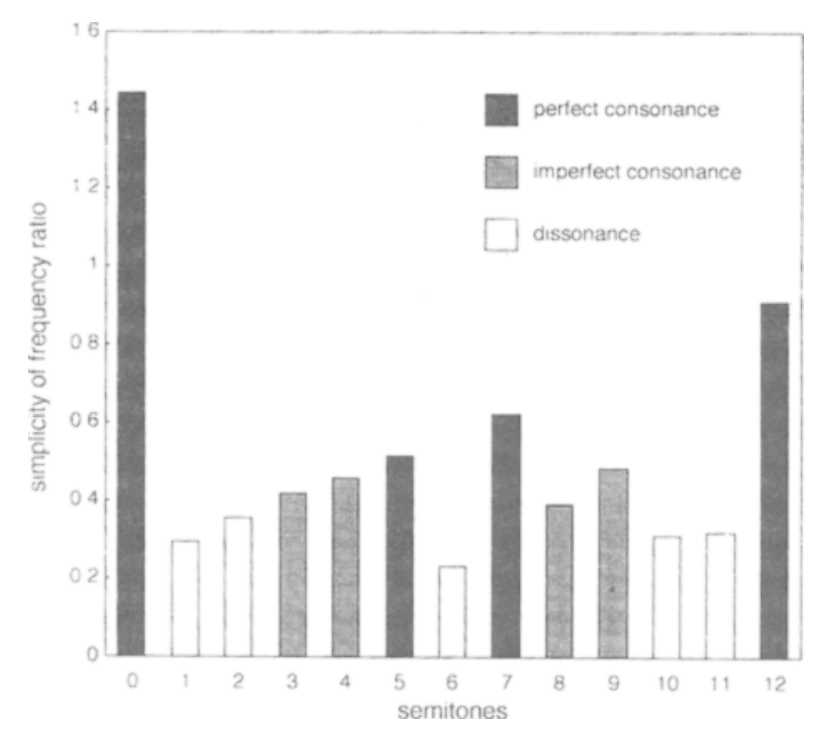

Figure 1. Quantified values of the simplicity of frequency ratios as a function of interval size (semitones) and Western music-theoretic classifications of consonance and dissonance. Higher values correspond to greater simplicity. 
(1990, 1992) considers the tritone to be essentially ambiguous, having the implications of either a small or a large interval, depending on its context. Similarly, music theory designates the tritone as an augmented fourth or a diminished fifth; both designations are considered unstable, requiring resolution to more stable intervals (Aldwell \& Schachter, 1989).

\section{RELATION TO OTHER MEASURES OF RATIO SIMPLICITY}

How does our index relate to other measures of the simplicity of frequency ratios? Using the larger of the two integers in a frequency ratio as their measure, van de Geer et al. (1962) also achieved mathematical simplicity and applicability to both pure and complex tones presented simultaneously or sequentially. There are notable differences, however. One is that van de Geer et al. calculated complexity rather than simplicity. Another is that van de Geer et al.'s measure yielded the same value (16) for justly tuned minor seconds and minor sevenths, compared with our value of .291 for the minor second and .311 (i.e., greater simplicity) for the minor seventh. Similarly, major thirds and sixths would be equally simple (a value of 5) for van de Geer et al., but somewhat different for our index (.455 for the major third vs. .481 for the major sixth). Despite the greater differentiation of our index, the values from the two measures were found to be highly but negatively correlated $(r=-829, N=23, p<$ .0001 , for the 23 intervals tested by van de Geer et al., 1962).
The only other direct measure of ratio simplicity, that of Levelt et al. (1966), is almost perfectly (negatively) correlated with our index for intervals with the same mean frequency. Indeed, for the 15 stimulus intervals presented by Levelt et al., all of which had a mean frequency of $500 \mathrm{~Hz}$, the two indexes yield a correlation coefficient of $r=-.927(N=15, p<.0001)$. Our index is considerably more useful, however, in that it is applicable to comparisons between intervals that are not equated for mean frequency (e.g., intervals with the same lower tone, say middle C). Moreover, because Levelt et al. computed the common logarithm (base 10) of the frequency (hertz) of the first harmonic common to two complex tones, the resulting index is not directly applicable to pure tones.

Mathematical estimates of the sensory consonance/ dissonance of two simultaneous complex tones can be considered indirect indexes of the simplicity of frequency ratios. (Sensory consonance is greater for simultaneous complex tones related by simple frequency ratios than by more complex frequency ratios, as noted above.) We examined associations between our index and Helmholtz's (1885/ 1954) measure of the degree of roughness, or dissonance, between two simultaneous complex tones. For the justly tuned intervals in Table 1 , the simplicity values were negatively correlated with the roughness values ( $r=$ $-.559, N=13, p<.05$ ). Standardized justly tuned roughness values and ratio-simplicity values as a function of interval size are shown in Figure 2 (with the sign of the standardized ratio-simplicity value reversed for purposes of comparison). The two values deviate most for the unison ( 0 semitones) and the minor second ( 2 semi-

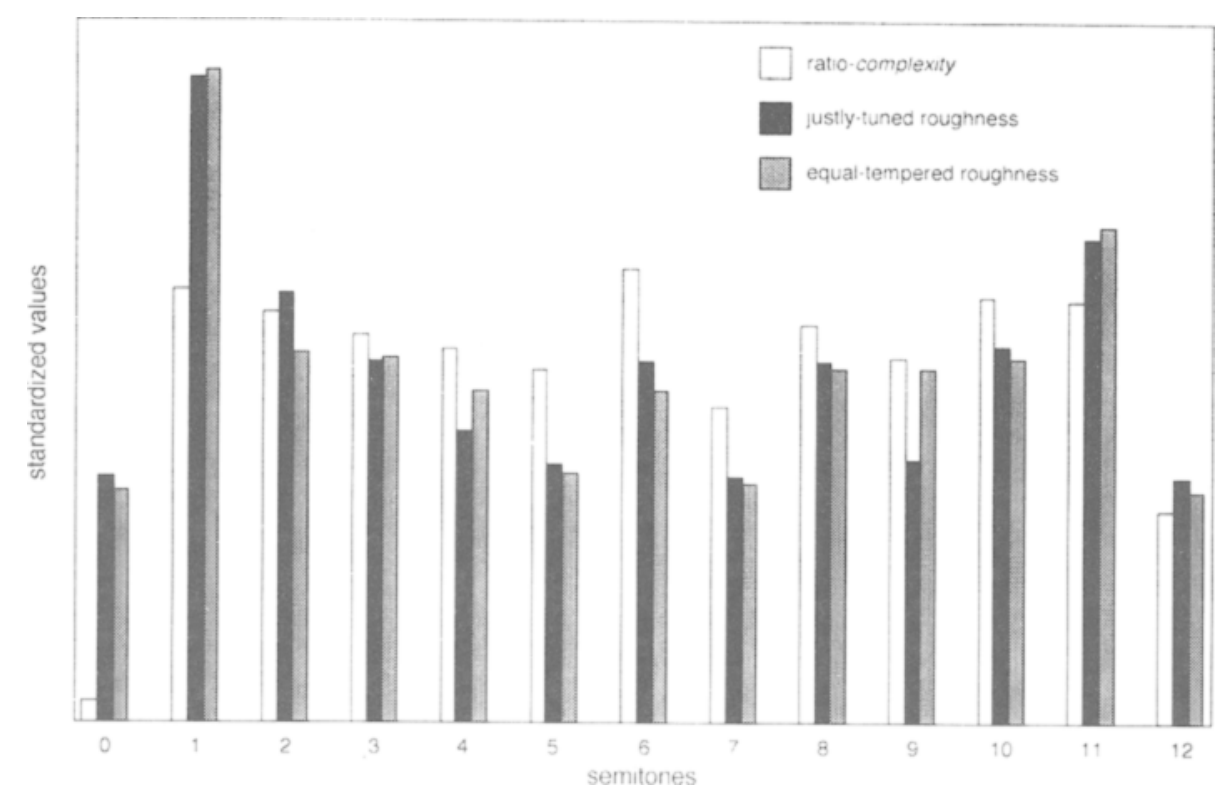

Figure 2. Standardized simplicity values of frequency ratios and standardized roughness values (Helmholtz, 1885/1954) for justly tuned and equal-tempered intervals as a function of interval size. For comparison purposes, the sign of the standardized simplicity values has been changed. Thus, higher values correspond to greater complexity and greater roughness. 
tones), which have considerably greater roughness values. Helmholtz (1885/1954) also calculated roughness values for equal-tempered intervals, which are mistuned from exact frequency ratios by up to $16 \%$ of 1 semitone. We also found these roughness values and our ratio-simplicity values (based on exact frequency ratios) to be negatively correlated $(r=-.576, N=13, p<.05)$. As can be seen in Figure 2, standardized equal-tempered values for roughness and simplicity are closely related for all intervals except for the unison and the minor second, as they are for justly tuned intervals. The finding of similar associations between simplicity and roughness values for both justly tuned and equal-tempered intervals supports the assumption that the values generated by our index of ratio simplicity are not uniquely tied to one tuning system.

Hutchinson and Knopoff (1978) incorporated the concept of critical bandwidth into their computation of the dissonance of simultaneous complex tones (see also Kameoka \& Kuriyagawa, 1969b). They calculated values for 1,500 intervals: 25 equal-tempered intervals ranging from 0 semitones (unisons) to 24 semitones (two octaves) at 60 different pitch positions for each interval (the lower tone of each interval ranged from $C_{1}$, or $32.7 \mathrm{~Hz}$, to $B_{5}$, or $987.8 \mathrm{~Hz}) .{ }^{1}$ Overall, smaller intervals had higher dissonance values than larger intervals (smaller intervals are more likely to have overlapping critical bands), and intervals with a lower mean frequency had higher dissonance values than intervals with a higher mean frequency (for frequencies under $500 \mathrm{~Hz}$, critical bandwidth increases in number of semitones as frequency decreases). We found the Hutchinson and Knopoff dissonance values to be negatively correlated with our simplicity values $(r=$ $-.406, N=1,500, p<.0001$ ). A multiple regression analysis was used to examine the predictive strength of the ratio-simplicity values, while controlling for interval size and mean pitch of interval (by including a predictor variable for both). The result was a highly significant fit to the dissonance values (multiple $R=.795, N=1,500$, $p<.0001$ ), with all three predictor variables making significant independent contributions $(p s<.0001)$.

\section{JUDGMENTS OF CONSONANCE AND DISSONANCE}

As a preliminary test of the generality of ratio simplicity as an explanatory construct, we examined the association between the simplicity of frequency ratios and judgments of consonance and dissonance. If simple frequency ratios confer processing advantages, then intervals with simpler ratios may be judged more consonant (i.e., more compatible, pleasant, smooth, etc.) than intervals with more complex ratios. The effect, if evident, should be present for patterns of pure or complex tones, and for listeners with or without musical training.

We compiled data on judgments of consonance and dissonance from several studies. For each study, or group of studies in the case of research preceding 1918, judgments of consonance and dissonance were rank ordered and compared with the rank ordering of ratio-simplicity values for intervals from 1 to 12 semitones. These values are presented in Table 2 together with rank-order correlations and probability values. The criteria for consonance and dissonance varied from study to study, as did the type of tones (pure or complex).

Malmberg (1918) reviewed 10 historical treatments of consonance and dissonance, from the 13 th century to the early 20 th century. For each of the 10 studies, he ranked the intervals from most to least consonant (Malmberg, 1918, Table 1, p. 103). The composite rank order of judgments presented in Table 2 was based on Krumhansl's (1990, Table 3.1, p. 57) averages of the ranks across the 10 studies. Malmberg also presented all possible pairs of the 12 harmonic (simultaneous) intervals (by means of piano or tuning fork) to musically trained listeners, who

Table 2

Rank Order of Ratio-Simplicity Values and Consonance Judgments Across Various Studies and Subject Groupings

\begin{tabular}{|c|c|c|c|c|c|c|c|c|c|c|c|c|c|c|c|}
\hline \multirow[b]{4}{*}{ Study } & & \multicolumn{12}{|c|}{ Interval Size (Semitones) } & \multirow[b]{4}{*}{$r_{s}$} & \multirow[b]{4}{*}{$p$} \\
\hline & \multirow[b]{3}{*}{ Subjects } & 12 & 7 & 5 & 9 & 4 & 3 & 8 & 2 & 11 & 10 & 1 & 6 & & \\
\hline & & \multicolumn{12}{|c|}{ Ratio-Simplicity Rank } & & \\
\hline & & 1 & 2 & 3 & 4 & 5 & 6 & 7 & 8 & 9 & 10 & 11 & 12 & & \\
\hline \multicolumn{16}{|l|}{ Malmberg (1918) } \\
\hline Piano & trained & 1 & 2 & 5 & 3 & 4 & 7 & 6 & 10 & 11 & 9 & 12 & 8 & .881 & $<.0005$ \\
\hline Tuning fork & trained & 1 & 2 & 4 & 3 & 5 & 7 & 6 & 11 & 10 & 9 & 12 & 8 & .888 & $<.0005$ \\
\hline \multicolumn{16}{|l|}{ Guernsey (1928) } \\
\hline \multirow[t]{3}{*}{ Smoothness } & untrained & 1 & 3 & 2 & 5 & 4 & 6 & 8 & 11 & 10 & 7 & 12 & 9 & .881 & $<.0005$ \\
\hline & mod. trained & 1 & 2 & 5 & 4 & 3 & 7 & 8 & 11 & 10 & 6 & 12 & 9 & .839 & $<.001$ \\
\hline & trained & 1 & 2 & 3 & 4 & 5 & 7 & 6 & 10 & 11 & 8 & 12 & 9 & .916 & $<.0001$ \\
\hline \multirow{3}{*}{ Pleasantness } & untrained & 4 & 5 & 3 & 1 & 2 & 6 & 7 & 11 & 10 & 8 & 12 & 9 & .790 & $<.005$ \\
\hline & mod. trained & 8 & 6 & 3 & 2 & 1 & 5 & 4 & 10 & 11 & 7 & 12 & 9 & .573 & $<.1$ \\
\hline & trained & 11 & 10 & 2 & 1 & 3 & 4 & 5 & 8 & 9 & 6 & 12 & 7 & .203 & n.s. \\
\hline \multirow{4}{*}{$\begin{array}{l}\text { Butler \& Daston } \\
\text { (1968) }\end{array}$} & untrained & & & & & & & & & & & & & & \\
\hline & U.S.A. & 5 & 2 & 6 & 4 & 1 & 8 & 3 & 7 & 11 & 10 & 12 & 9 & .734 & $<.01$ \\
\hline & Japan (West.) & 4 & 1 & 2 & 6 & 3 & 7 & 5 & 8 & 12 & 10 & 11 & 9 & .853 & $<.0005$ \\
\hline & Japan (Trad.) & 5 & 3 & 2 & 7 & 1 & 6 & 4 & 8 & 12 & 10 & 11 & 9 & .755 & $<.005$ \\
\hline
\end{tabular}

Note- - mod. = moderately; West. $=$ Western; Trad. $=$ traditional. 
were asked to judge which interval was more smooth, pure, and well blended. He calculated two composite scores (one for each timbre), from the three judgments for each interval, on the basis of how many times it was chosen over the other 11 intervals. The rank orders of these scores are presented separately for both timbres (see Table 2).

In an attempt to determine whether Malmberg's (1918) findings were independent of musical training, Guernsey (1928) conducted a similar experiment with listeners who were musically trained, moderately trained, or untrained. She presented (with pure-tone resonators) the 12 intervals used by Malmberg to the three groups of listeners. In one experiment, the listeners provided smoothness judgments; in another, they provided pleasantness judgments. The rank orders of intervals, based on smoothness and pleasantness judgments, are listed in Table 2 for each group of subjects.

Butler and Daston (1968) examined the generality of Malmberg's (1918) results across cultures by testing college students from the United States and Japan. The Japanese students were subdivided into two groups: those who preferred Western music and those who preferred traditional Japanese music. All the listeners made pairwise preference judgments of the 12 intervals, which were presented on an organ (complex tones). The rank orders of interval preferences for each group are listed in Table 2 .

Rank-order (Spearman) correlations (Table 2) show remarkable agreement between interval judgments and the relative simplicity of frequency ratios across the various studies and subject groupings. In fact, strong associations prevailed across different cultural backgrounds, musical experience, judgment criteria (preference or smoothness), and tones (pure or complex). Ratio simplicity was less successful in predicting pleasantness judgments (Guernsey, 1928), particularly for listeners with more musical training. This finding may reflect the influence of Western harmonic structure (i.e., the predominance of thirds and sixths with their moderately simple frequency ratios) on listeners with more musical training. Thus, extensive training in Western music may increase culturally biased responding, thereby decreasing effects of ratio simplicity. Higher evaluations of moderately simple frequency ratios (i.e., imperfect consonances) compared with very simple frequency ratios (i.e., perfect consonances) have also been reported by van de Geer et al. (1962).

Krumhansl and Kessler (1982) required musically trained listeners to rate how well a test tone fit with a musical key, establishing the key by playing a scale, chord, or cadence (a combination of chords signaling the end of a musical phrase). Because musical keys have a tonic note, which acts as a perceptual reference point, such ratings also reflect the consonance of the interval between the tonic and the test tone. To minimize the influence of pitch distance, Krumhansl and Kessler used special tones with poorly defined pitch height (see Shepard, 1964). They derived two hierarchies of stability values (a continuous mea- sure)-one for tones in major keys, and the other for tones in minor keys. Ratio simplicity was a significant predictor of the hierarchy of values for both major and minor keys (major keys, $r=.862, N=13, p<.0005$; minor keys, $r=.790, N=13, p<.005)$. Thus, regardless of whether a musical key is in a major or minor mode, tones are perceived to fit better in the key when they form simple frequency ratios with the tonic of that key than when they form more complex ratios. Moreover, the association between major and minor hierarchy values was weaker $(r=.651, N=13, p<.05)$ than the association of either hierarchy with the ratio-simplicity index.

\section{DISCRIMINATION OF TONE SEQUENCES}

The current claim of processing advantages for simple frequency ratios arose from performance on tasks in which listeners were required to discriminate changes in interval size (Schellenberg, 1994; Schellenberg \& Trehub, in press; Trainor \& Trehub, 1993a, 1993b). Changes from simple ratios to more complex ratios were found to be more readily detectable than changes from complex to simpler ratios. This pattern of asymmetries implies that intervals with simple ratios are encoded more efficiently than are intervals with more complex ratios, providing a differentially effective basis for the detection of changes. Similar asymmetries have been reported for the detection of changes to conventionally structured and unconventionally structured linguistic and nonlinguistic patterns (Bharucha, Olney, \& Schnurr, 1985; Bharucha \& Pryor, 1986).

\section{Adult and Child Listeners}

Schellenberg and Trehub's (in press) listeners, adults with little musical training, heard a standard five-tone pattern (two component tones in an alternating sequence) repeating in transposition (same frequency ratio between component tones, different absolute frequencies), and were asked to indicate when a comparison pattern (different frequency ratio, different absolute frequencies) was substituted for the standard. The standard and comparison patterns were comprised of perfectly consonant intervals (frequency ratio of $3: 2$ or $4: 3$ ), imperfectly consonant intervals $(5: 4$ or $8: 5)$, or a dissonant interval (45:32). The effect of ratio simplicity was graded; our reanalysis of the data showed that performance improved as the ratio between tones in the standard pattern became simpler $(r=.486, N=40, p<.005)$ and as the ratio between tones in the comparison pattern became more complex $(r=-.536, N=40, p<.001)$.

Schellenberg (1994) found comparable perceptual asymmetries as a function of ratio simplicity when they used a different methodology (same/different) with adults and 6-year-old children. Performance was more accurate when the standard interval (presented first) had a simple frequency ratio $(2: 1,3: 2,4: 3)$ and the comparison interval (presented second) had a more complex ratio $(15: 8,32: 15$, $45: 32$ ) than when the simple ratio followed the complex ratio. The processing advantage for simple frequency 
ratios was evident even in 6-year-old listeners with little or no musical training. For all the listeners, moreover, performance did not differ from chance levels when the standard interval had a complex ratio. These results are consistent with the view that tones related by simple ratios are inherently easier to process than tones related by more complex ratios. Comparable findings with infant listeners would constitute stronger evidence in this regard.

\section{Infant Listeners}

If processing advantages associated with simple frequency ratios are largely independent of musical enculturation, then such advantages should be evident in infancy. An analysis of relevant infant discrimination data was made possible by the cooperation of Trainor (1991), who tested 40 infants 9 to 11 months of age with a procedure similar to that used by Schellenberg and Trehub (in press). Trainor's (1991, chap. 3) infants were trained to turn their heads toward a loudspeaker when a comparison pattern was substituted for the standard. One of her standard patterns exemplified a structure considered basic to Western tonal music (the major triad), consisting of the first, third, and fifth notes of the major scale, ascending and descending (e.g., $\mathrm{C}_{4}-\mathrm{E}_{4}-\mathrm{G}_{4}-\mathrm{E}_{4}-\mathrm{C}_{4}$ ). The other standard pattern (e.g., $\mathrm{C}_{4}-\mathrm{E}_{4}-\mathrm{G}_{4}-\mathrm{E}_{4}-\mathrm{C}_{4}$, the augmented triad) was similar in configuration (rise-fall contour) and pitch range, but much less conventional in structure-its note set did not belong to any single major scale. Comparison patterns were formed by displacing the highest tone of the pattern upward or downward (from an exact transposition) by a semitone. In line with earlier findings (Cohen et al., 1987), Trainor found superior infant discrimination when the standard pattern was conventionally structured. In further research (Trainor \& Trehub, 1993b), the source of the processing advantage was identified as the relation between the low and high tones (e.g., $\mathrm{C}_{4}$ and $\mathrm{G}_{4}$ ). Sensitivity to the contour of tone sequences (Chang \& Trehub, 1977; Ferland \& Mendelson, 1989; Trehub, Bull, \& Thorpe, 1984; Trehub, Thorpe, \& Morrongiello, 1985, 1987) may have drawn the infants' attention to the locus of contour change (the high tone) as well as to the first and last tones (the low tones), enhancing their sensitivity to relations between the low and high tones of Trainor's (1991) patterns.
We reanalyzed Trainor's (1991) data as a function of the simplicity of frequency ratios between low and high tones of the standard and comparison patterns. Frequency ratios are listed in Table 3 for each of Trainor's four conditions. Mean $d^{\prime}$ scores and standard deviations are also presented. As predicted, the infants performed better when the low and high tones of the standard pattern were related by a simple ratio (perfect fifth, ratio of 3:2) than by a more complex ratio (minor sixth, ratio of $8: 5$ ) $[t(38)=3.80, p<.001]$. Performance also improved as the ratio between the low and high tones of the comparison pattern became more complex; the correlation between the infants' discrimination scores and the ratio simplicity of the comparison pattern was $r=-.737(N=$ $40, p<.0001$ ). If performance varied as a function of the simplicity difference between the standard and comparison patterns, then the infants should have been most accurate on the simplest standard and most complex comparison pattern (i.e., major triad-downward condition), least accurate on the most complex standard and simplest comparison pattern (i.e., augmented triad-downward condition), and intermediate on the other two conditions. As can be seen in Table 3, the pattern of discrimination scores is consistent with this prediction. Indeed, we found the infants' performance to be a function of the difference in ratio-simplicity values between standard and comparison patterns $(r=.670, N=40, p<.0001)$. Thus, the pattern of results for infant listeners is remarkably consistent with that reported for children and adults (Schellenberg, 1994; Schellenberg \& Trehub, in press), suggesting that advantages for simple over more complex ratios are independent of musical enculturation.

\section{JUDGMENTS OF SIMILARITY}

If the discriminability of intervals is related to differences in their ratio simplicity, then the perceived similarity of intervals may be affected by similarities in ratio simplicity. It is unclear, however, whether tones perceived to be compatible (i.e., the component tones of intervals with simple ratios) would also be perceived as more similar than tones perceived to be incompatible (i.e., the component tones of intervals with complex ratios). In any case, pitch distance is likely to play a critical role, with listeners perceiving tones closer in pitch as more similar than tones

Table 3

Mean Discrimination Scores $\left(d^{\prime}\right)$ and Standard Deviations as a Function of the Frequency Ratio Between Low and High Tones of Standard and Comparison Patterns (Trainor, 1991)

\begin{tabular}{lccrrr}
\hline \multicolumn{1}{c}{ Condition } & \multicolumn{2}{c}{ Frequency Ratio } & & \\
\cline { 2 - 3 } \multicolumn{1}{c}{ Standard } & Comparison & Mean $d^{\prime}$ & $S D$ \\
\hline Major-upward change & $3: 2$ & $8: 5$ & 0.50 & 0.23 \\
Major-downward change & $3: 2$ & $45: 32$ & 0.89 & 0.43 \\
Augmented-upward change & $8: 5$ & $5: 3$ & 0.47 & 0.51 \\
Augmented-downward change & $8: 5$ & $3: 2$ & -0.28 & 0.38 \\
\hline
\end{tabular}


further apart, and intervals nearer in size (pitch distance between tones) as more similar than intervals with greater size differences.

\section{Similarity Between Tones}

Krumhansl (1979, Experiment 1) presented musically trained adults with a key-defining tonal pattern (i.e., an ascending or descending $\mathrm{C}$-major scale or a $\mathrm{C}$-major chord) followed by two successive test tones taken from the set of 13 equal-tempered tones in the same octave as that for the key-defining pattern. Listeners rated how similar the first test tone was to the second (in the musical key suggested by the context) by providing ratings for each pair of tones (78 pairs) presented in both possible orders (a total of 156 ratings). If the simplicity of frequency ratios influenced highly trained listeners, then higher ratings would be expected for tones related by simple ratios (e.g., perfect fifths: $\mathrm{C}_{4}-\mathrm{G}_{4}, \mathrm{G}_{4}-\mathrm{C}_{4}, \mathrm{C}{ }_{4}-\mathrm{G}_{4}, \mathrm{G} \sharp_{4}-\mathrm{C}_{4}$, etc.) than for tones related by more complex ratios (e.g., tritones: $E_{4}-A H_{4}, A H_{4}-E_{4}, F_{4}-B_{4}, B_{4}-F_{4}$, etc.), irrespective of the influence of the tonal context, the order of the test tones, and differences in the relative stability between test tones in the established context. Because Krumhansl's (1979) experiment was designed to tap the influence of a well-defined tonal context (i.e., the key of $\mathrm{C}$ major), it is a conservative test of the ratio-simplicity hypothesis, which is independent of tonal context.

We also examined variation in the similarity ratings as a function of pitch distance and ratio simplicity. Following Krumhansl (1979, Figure 1), pairs of identical tones (i.e., the 13 possible unisons in an octave range) were assumed to have a maximal similarity value of 7 (increasing the total number of intervals to 169). As expected, listeners gave lower similarity ratings to tones that were more distant in pitch (in number of semitones; $r=-.502$, $N=169, p<.0001$ ). Ratio-simplicity values were strongly associated with similarity ratings for pairs of tones $(r=.714, N=169, p<.0001)$, indicating that tones related by simpler ratios were judged to be more similar than tones related by more complex ratios. In a multiple regression analysis that controlled for effects of pitch distance, similarity ratings were modeled as a function of four predictor variables: (1) ratio simplicity between the two test tones, (2) ratio simplicity between the first test tone and the tonic, (3) ratio simplicity between the second test tone and the tonic, and (4) pitch distance in semitones between the two test tones. The fit of the model was highly significant (multiple $R=.846, N=$ $169, p<.0001$ ), and all four predictors made significant independent contributions $(p s<.0001)$. The results from the multiple regression analysis are provided in $\mathrm{Ta}-$ ble 4 (psychoacoustic model). Similarity ratings were, in general, higher when frequency ratios were simpler as opposed to more complex, and they were higher when the test tones were closer in pitch. Thus, two psychoacoustic factors, ratio simplicity and pitch distance, provide a clear and parsimonious explanation of the variation in Krumhansl's (1979) results.
Table 4

Three Models Fit to Similarity Data From Krumhansl (1979, Table 2)

\begin{tabular}{|c|c|c|c|}
\hline Predictor Variable & $d f$ & $F$ & $p$ \\
\hline \multicolumn{4}{|c|}{$\begin{array}{l}\text { Psychoacoustic Model } \\
\text { (Multiple } R=.846, N=169, p<.0001 \text { ) }\end{array}$} \\
\hline \multicolumn{4}{|l|}{ Frequency ratio } \\
\hline Between test tones & 1 & 169.0 & $<.00$ \\
\hline Between first test tone and tonic & 1 & 16.19 & $<.000$ \\
\hline Between second test tone and tonic & 1 & 38.92 & $<.000$ \\
\hline Pitch distance between test tones & 1 & 94.20 & $<.00$ \\
\hline \multicolumn{4}{|c|}{$\begin{array}{l}\text { Music-Theoretic Model } \\
\text { (Multiple } R=.845, N=169, p<.0001 \text { ) }\end{array}$} \\
\hline Tonality of first test tone & 2 & 22.28 & $<.000$ \\
\hline Tonality of second test tone & 2 & 57.04 & $<.00$ \\
\hline Tonality of first $\times$ second test tone & 4 & 33.56 & $<.000$ \\
\hline Pitch distance between test tones & 1 & 158.4 & $<.00$ \\
\hline \multicolumn{4}{|c|}{$\begin{array}{c}\text { Combined Model } \\
\text { (Multiple } R=.930, N=169, p<.0001 \text { ) }\end{array}$} \\
\hline Tonality of first test tone & 2 & 32.38 & $<.000$ \\
\hline Tonality of second test tone & 2 & 95.29 & $<.000$ \\
\hline Tonality of first $\times$ second test tone & 4 & 18.54 & $<.000$ \\
\hline Pitch distance between test tones & 1 & 193.4 & $<.000$ \\
\hline Frequency ratio between test tones & 1 & 176.5 & $<.00$ \\
\hline
\end{tabular}

Krumhansl's (1979) explanation was primarily based on the stability of test tones within a musical key. Each test tone was considered to be in one of three tonal categories: the tonic triad of the implied key (stable tones), the scale of the implied key but not the tonic triad (moderately stable tones), and nondiatonic (unstable) tones. Because the order of the test tones and pitch distance also influenced listeners' ratings, Krumhansl's (1979) "model" of the variation in her data was assumed to be a $3 \times 3$ analysis of covariance, with three levels of the tonal category of the first test tone, three levels of the tonal category of the second tone, and a covariate representing the distance in pitch between test tones. Results from an analysis based on this model are provided in Table 4 (music-theoretic model). The resulting multiple $R$ was $.845(N=169, p<.0001)$, virtually identical to the psychoacoustic model in goodness of fit. Thus, the performance of musically trained listeners on a musically relevant task can be explained as effectively with psychoacoustic factors as it can with culture-specific, musictheoretic factors.

The psychoacoustic model predicts that, in general, tones closer in pitch and those related by a simple ratio will be rated as more similar than tones more distant in pitch and those related by a more complex ratio. By contrast, the music-theoretic model accounts for the data by way of pitch distance and the stability of each test tone in relation to the established key. The addition of a predictor variable (to the music-theoretic model) for ratio simplicity between test tones significantly improved the fit to the data $[F(1,158)=176.5, p<.0001]$, generating a multiple $R$ of $.930(N=169, p<.0001)$. Results 
from this analysis are shown in Table 4 (combined model). Thus, tones related by simple ratios were judged to be more similar than tones related by more complex ratios, even with the influence of tonal harmonic factors and pitch distance held constant. Effects of ratio simplicity above and beyond those associated with music-theoretic constructs provide compelling evidence of the influence of frequency ratios on the perception of tone patterns.

\section{Similarity Between Intervals}

If simplicity of frequency ratios functions as a psychoacoustic primitive, then it may contribute to the perceived similarity between intervals. Levelt et al. (1966) had listeners judge similarities among 15 different complextone intervals and 15 different pure-tone intervals. Multidimensional scaling revealed a three-dimensional solution for complex-tone judgments. The coordinates of two dimensions formed a U-shaped curve corresponding to distance in pitch (interval size). The coordinates of the third dimension of the solution were associated with ratio simplicity $(r=.826, N=15, p<.0005)$, indicating its influence on similarity judgments, as proposed above. For complex tones, then, judgments of interval similarity were based on pitch distance (interval size) and ratio simplicity, as were judgments of tone similarity (Krumhansl, 1979). In the three-dimensional scaling solution for pure-tone intervals, no dimension was significantly correlated with ratio simplicity. Coordinates from two dimensions formed the same U-shaped curve based on interval size that was found with complex tones. However, the third dimension indicated the presence of reference points associated with simple frequency ratios, with the perfect fourth (4:3) and major third (5:4) at a local minimum, the perfect fifth (3:2) at a local maximum, and the octave $(2: 1)$ at another local minimum (Levelt et al., 1966, Figure 2). The coordinates of this dimension could not be predicted by our index because intervals with simple ratios had either very high or very low values. Nevertheless, effects of ratio simplicity on similarity judgments for pairs of intervals (pure or complex tones) or tones confirm the applicability of the construct to perceived similarity.

\section{DISCUSSION}

We have reported effects of simplicity of frequency ratios on the perception of pure and complex tones for patterns involving sequential as well as simultaneous intervals. Ratio simplicity accounted for judgments of both consonance and similarity across a wide range of tasks and listeners. It also accounted for the ability of listeners to distinguish one tone sequence from another, whether they were musically experienced adults or 9-month-old infants. These findings are consistent with the hypothesis that tones related by simple frequency ratios are processed more readily than tones related by more complex ratios.
Our finding that ratio simplicity affects perception across tasks, listeners, and contexts is inconsistent with the prevailing view that the relation between simple frequency ratios and auditory pattern processing is a product of enculturation (e.g., Burns \& Ward, 1982; Dowling \& Harwood, 1986). Closer inspection of this literature reveals, however, that the likely source of the apparent discrepancies is methodological. Although Plomp and Levelt (1965) reported no effects of ratio simplicity on judgments of consonance (simultaneous pure tones), their exclusion of common, familiar (consonant) intervals resulted in the absence of stimuli with simple frequency ratios, obscuring potential effects of ratio simplicity. Kameoka and Kuriyagawa (1969a), who found very small peaks in consonance judgments for simple frequency ratios, had listeners compare intervals with a very narrow range of consonance (i.e., ratio simplicity) differences, thereby minimizing the effects of ratio simplicity. Thus, the findings of Plomp and Levelt and those of Kameoka and Kuriyagawa reveal little about how simplicity of frequency ratios, in general, relates to perceived consonance and dissonance.

Anomalies in studies of intervallic similarity (e.g., octave equivalence) may also have methodological origins, especially the failure to find octave effects in adult listeners (Allen, 1967; Kallman, 1982), given their presence in infant listeners (Demany \& Armand, 1984). The finding that musically untrained adults based their judgments of tone similarity solely on pitch distance may stem from the use of a very wide pitch range (four octaves in Allen, 1967; 28 semitones in Kallman, 1982), which increased the salience of pitch differences. Very high tones are obviously unlike very low tones. Indeed, narrowing the pitch range of intervals to be judged resulted in the emergence of some effects of octave equivalence (Kallman, 1982). Finally, Burns and Ward's (1978) finding of the apparent irrelevance of simple frequency ratios to perceptual judgments may be attributable to the excessive difficulty of their task. They asked musically untrained listeners to identify which of two intervals was wider, raising the possibility that subjects who were unable to perform this unusual task may have been able to differentiate the intervals from one another. In fact, 2 of the 6 untrained listeners had to be excluded from the data analysis because they consistently judged the relative width of intervals by the absolute pitch of their initial tone.

Despite the aforementioned failures to find effects of ratio simplicity, the effects of simplicity on perceptual processing are, by and large, pervasive. Definitive causal evidence regarding the association between frequency ratios and scale structure is still unavailable. Nevertheless, there is a plausible direction of influence despite the contention that the association is simply a coincidence (e.g., Dowling \& Harwood, 1986)-a "remarkable" coincidence, according to Aldwell and Schachter $(1989$, p. 26). This coincidence has also been explained by the degree of sensory consonance of simultaneous complex tones, 
for which the alignment of adjacent harmonics (and hence frequency ratios) are a contributing factor (Burns \& Ward, 1982). Thus, although processing advantages for simple ratios could indeed influence scale structure, the universality of simple ratios in scales does not constitute definitive evidence of such processing advantages.

Our primary objective, in the present review, was to demonstrate that simplicity of frequency ratios provides a parsimonious account of the available data on interval perception. We can offer no explanations-only speculations-about why simple frequency ratios may confer processing advantages for inexperienced as well as experienced listeners.

A number of investigators (e.g., Boomsliter \& Creel, 1961; Patterson, 1986; Roederer, 1973) maintain that information in the temporal pattern of neural discharge (specifically, similarity) provides the clue to interval preferences and consonance. According to Moore (1989), temporal firing patterns also could account for similarities between individual tones related by simple frequency ratios and for our inability to perceive melody or musical pitch at frequencies above $5000 \mathrm{~Hz}$ (Attneave \& Olson, 1971), where neural synchrony is not operative. This general approach is compatible with the claim of inherent ease of processing for tones related by simple frequency ratios (Cohen et al., 1987; Schellenberg \& Trehub, in press; Trehub \& Trainor, 1993).

The data presented in this paper are also consistent with Terhardt's $(1974,1978,1984)$ contention that, from birth, exposure to naturally occurring sounds (especially speech) leads to familiarity with the intervals between audible (lower) harmonics, which exemplify simple frequency ratios $(2: 1,3: 2,4: 3$, etc.). For this approach to be applicable to the infant discrimination data, however, the requisite learning would have to occur in the early months of life. Indeed, there is evidence of early recognition of the mother's voice (DeCasper \& Fifer, 1980; Mehler, Bertoncini, Barrière, \& Jassik-Gerschenfeld, 1978) and of perceptual effects of language of exposure by 6 months of age (Kuhl, Williams, Lacerda, Stevens, \& Lindblom, 1992; Polka \& Werker, 1994). Experiential effects such as these could be considered to exemplify innately guided learning (Gould \& Marler, 1987), a process characterized by initial selectivity in responsiveness and rapid learning.

It is tempting to describe simultaneous and sequential tones with simple frequency ratios as exhibiting good form, particularly in light of their apparent processing advantages. Although patterns of simultaneous tones may meet conventional Gestalt (e.g., Koffka, 1935; Köhler, 1947) criteria of phenomenal simplicity (e.g., sounding simpler than tones with complex ratios, sounding fused or "whole") or information-theoretic criteria of pattern goodness (e.g., Garner, 1970, 1974; Pomerantz \& Kubovy, 1981), patterns of sequential tones do not (but see Bartlett, 1993). Instead, such patterns meet a different but equally stringent test of goodness-ease of processing by naive listeners (Trehub \& Trainor, 1993).
Tones related by simple frequency ratios may constitute natural intervals or prototypes (Rosch, 1975), providing a perceptual frame of reference for other intervals (Trehub \& Unyk, 1991). Their contribution to grouping processes (Deutsch, 1982; McAdams, 1993) and to the analysis of complex auditory events or "scenes" (Bregman, 1990,1993 ) remains to be determined.

\section{REFERENCES}

AldWell, E., \& SCHACHTER, C. (1989). Harmony and voice leading (2nd ed.). San Diego: Harcourt Brace Jovanovich.

Allen, D. (1967). Octave discriminability of musical and non-musical subjects. Psychonomic Science, 7, 421-422.

Attneave, F., \& Olson, R. K. (1971). Pitch as a medium: A new approach to psychophysical scaling. American Journal of Psychology, 84, 147-166.

BartletT, J. C. (1993). Tonal structure of melodies. In T. J. Tighe \& W. J. Dowling (Eds.), Psychology and music: The understanding of melody and rhythm (pp. 39-61). Hillsdale, NJ: Erlbaum.

Bharucha, J. J., Olney, K. L., \& Schnurr, P. P. (1985). Detection of coherence-disrupting and coherence-conferring alterations in text. Memory \& Cognition, 13, 573-578.

Bharucha, J. J., \& PrYoR, J. H. (1986). Disrupting the isochrony underlying rhythm: An asymmetry in discrimination. Perception \& Psychophysics, 40, 137-141.

Boomsliter, P., \& Creel, W. (1961). The long pattern hypothesis in harmony and hearing. Journal of Music Theory, 5, 2-31.

Bregman, A. S. (1990). Auditory scene analysis. Cambridge, MA: MIT Press.

Bregman, A. S. (1993). Auditory scene analysis: Hearing in complex environments. In S. McAdams \& E. Bigand (Eds.), Thinking in sound: The cognitive psychology of human audition (pp. 10-36). Oxford: Oxford University Press.

BURNS, E. M., \& WARD, W. D. (1978). Categorical perceptionphenomenon or epiphenomenon: Evidence from experiments in the perception of melodic musical intervals. Journal of the Acoustical Society of America, 63, 456-468.

BurNS, E. M., \& WARD, W. D. (1982). Intervals, scales, and tuning. In D. Deutsch (Ed.), The psychology of music (pp. 241-269). New York: Academic Press.

Butler, J. W., \& Daston, P. G. (1968). Musical consonance as musical preference: A cross-cultural study. Journal of General Psychology, 79, 129-142.

Chang, H. W., \& Trehub, S. E. (1977). Auditory processing of relational information by young infants. Journal of Experimental Child Psychology, 24, 324-331.

Cohen, A. J., Thorpe, L. A., \& Trehub, S. E. (1987). Infants' perception of musical relations in short transposed tone sequences. Canadian Journal of Psychology, 41, 33-47.

DeCasper, A. J., \& Fifer, W. P. (1980). Of human bonding: Newborns prefer their mothers' voices. Science, 208, 1174-1176.

Demany, L., \& Armand, F. (1984). The perceptual reality of tone chroma in early infancy. Journal of the Acoustical Society of America, 76, 57-66.

DEUTSCH, D. (1982). Grouping mechanisms in music. In D. Deutsch (Ed.), The psychology of music (pp. 99-134). New York: Academic Press.

Dowling, W. J., \& Harwood, D. L. (1986). Music cognition. San Diego: Academic Press.

Ferland, M. B., \& Mendelson, M. J. (1989). Infants' categorization of melodic contour. Infant Behavior \& Development, 12, 341-355.

GARNER, W. R. (1970). Good patterns have few alternatives. American Scientist, 58, 34-42.

GARNER, W. R. (1974). The processing of information and structure. Hillsdale, NJ: Erlbaum.

Gould, J. L., \& Marler, P. (1987). Learning by instinct. Scientific American, 256, 74-85. 
GUERNSEY, M. (1928). The role of consonance and dissonance in music. American Journal of Psychology, 40, 173-204.

HELMHOLTZ, H. L. F. voN (1954). On the sensations of tone as a physiological basis for the theory of music (rev. ed., A. J. Ellis, Ed. and Trans.). New York: Dover. (Original work published 1885)

Huron, D., \& Sellmer, P. (1992). Critical bands and the spelling of vertical sonorities. Music Perception, 10, 129-150.

Hutchinson, W., \& KNopoff, L. (1978). The acoustic component of Western consonance. Interface, 7, 1-29.

Kallman, H. J. (1982). Octave equivalence as measured by similarity ratings. Perception \& Psychophysics, 32, 37-49.

KameoKa, A., \& Kuriyagawa, M. (1969a). Consonance theory: Part I. Consonance of dyads. Journal of the Acoustical Society of America, 45, 1451-1459.

Kameoka, A., \& Kuriyagawa, M. (1969b). Consonance theory: Part II. Consonance of complex tones and its calculation method. Jour nal of the Acoustical Society of America, 45, 1460-1469.

KoffKA, K. (1935). Principles of Gestalt psychology. London: Routledge \& Kegan Paul.

KöHLER, W. (1947). Gestalt psychology: An introduction to new concepts of modern psychology. New York: Liveright.

KRUMHANSL, C. L. (1979). The psychological representation of musical pitch in a tonal context. Cognitive Psychology, 11, 346-374.

KrumhansL, C. L. (1990). Cognitive foundations of musical pitch. New York: Oxford University Press.

Krumhansl, C. L., \& Kessler, E. J. (1982). Tracing the dynamic changes in perceived tonal organization in a spatial representation of musical keys. Psychological Review, 89, 334-368.

Kuhl, P. K., Williams, K. A., Lacerda, F., Stevens, K. N., \& LiNDBLOM, B. (1992). Linguistic experience alters phonetic perception in infants by 6 months of age. Science, 255, 606-608.

LERDAHL, F., \& JACKENDOFF, R. (1983). A generative theory of tonal music. Cambridge, MA: MIT Press.

Levelt, W. J. M., van de Geer, J. P., \& Plomp, R. (1966). Triadic comparisons of musical intervals. British Journal of Mathematical \& Statistical Psychology, 19, 163-179.

Malmberg, C. F. (1918). The perception of consonance and dissonance. Psychological Monographs, 25, 93-133.

MCADAms, S. (1993). Recognition of sound sources and events. In S. McAdams \& E. Bigand (Eds.), Thinking in sound: The cognitive psychology of human audition (pp. 146-198). Oxford: Oxford University Press.

Mehler, J., Bertoncini, J., Barrière, M., \& Jassik-Gerschenfeld, D. (1978). Infant recognition of mother's voice. Perception, 7, 491-497.

MEYER, L. B. (1956). Emotion and meaning in music. Chicago: University of Chicago Press.

MoORE, B. C. J. (1989). An introduction to the psychology of hearing (3rd ed.). London: Academic Press.

NARMOUR, E. (1990). The analysis and cognition of basic melodic structures: The implication-realization model. Chicago: University of Chicago Press.

NARMOUR, E. (1992). The analysis and cognition of melodic complexity: The implication-realization model. Chicago: University of Chicago Press.

Patterson, R. D. (1986). Spiral detection of periodicity and the spiral form of musical scales. Psychology of Music, 14, 44-61.

Plomp, R. (1964). The ear as a frequency analyzer. Journal of the Acoustical Society of America, 36, 1628-1636.

Plomp, R. , LeVelT, W. J. M. (1965). Tonal consonance and critical bandwidth. Joumal of the Acoustical Society of America, 38, 548-560.

Polka, L., \& Werker, J. F. (1994). Developmental changes in the perception of nonnative vowel contrasts. Journal of Experimental Psychology: Human Perception \& Performance, 20, 421-435.

Pomerantz, J. R., \& Kubovy, M. (1981). Perceptual organization: An overview. In M. Kubovy \& J. R. Pomerantz (Eds.), Perceptual organization (pp. 423-456). Hillsdale, NJ: Erlbaum.
RAKOWSKI, A. (1990). Intonation variants of musical intervals in isolation and in musical contexts. Psychology of Music, 18, 60-72.

RameAu, J. P. (1971). Treatise on hamony (P. Gosseth, Trans.). New York: Dover. (Original work published 1722)

ROEDERER, J. (1973). Introduction to the physics and psychophysics of music. Berlin: Springer-Verlag.

Rosch, E. (1975). Cognitive reference points. Cognitive Psychology, 7, 532-547.

SACHS, C. (1943). The rise of music in the ancient world: East and West. New York: Norton.

SCHELLENBERG, E. G. (1994). Effects of frequency ratio simplicity on the perception of tone patterns. Unpublished doctoral dissertation, Cornell University, Ithaca, NY.

Schellenberg, E. G., \& Trehub, S. E. (in press). Frequency ratios and the discrimination of pure tone sequences. Perception \& Psychophysics.

SHEPARD, R. N. (1964). Circularity in judgments of relative pitch. Journal of the Acoustical Society of America, 36, 2346-2353.

TerhardT, E. (1974). Pitch, consonance, and harmony. Journal of the Acoustical Society of America, 55, $1061-1069$.

TERHARDT, E. (1978). Psychoacoustic evaluation of musical sounds. Perception \& Psychophysics, 23, 483-492.

TERHARDT, E. (1984). The concept of musical consonance: A link between music and psychoacoustics. Music Perception, 1, 276-295.

TraINOR, L. J. (1991). The origins of musical pattern perception: $A$ comparison of infants' and adults' processing of melody. Unpublished doctoral dissertation, University of Toronto.

Trainor, L. J., \& Trehub, S. E. (1993a). Musical context effects in infants and adults: Key distance. Joumal of Experimental Psychology: Human Perception \& Performance, 19, 615-626.

TrainoR, L. J., \& TrehUB, S. E. (1993b). What mediates infants' and adults' superior processing of the major over the augmented triad? Music Perception, 11, 185-196.

TrehuB, S. E., Bull, D., \& ThORPE, L. A. (1984). Infants' perception of melodies: The role of melodic contour. Child Development, 55, 821-830.

Trehub, S. E., Thorpe, L. A., \& Morrongiello, B. A. (1985). Infants' perception of melodies: Changes in a single tone. Infant Behavior \& Development, 8, 213-223.

Trehub, S. E., Thorpe, L. A., \& Morrongiello, B. A. (1987). Organizational processes in infants' perception of auditory patterns. Child Development, 58, 741-749.

Trehub, S. E., ThORPe, L. A., Trainor, L. J. (1990). Infants' perception of good and bad melodies. Psychomusicology, 9, 5-15.

TreHuB, S. E., \& Trainor, L. J. (1993). Listening strategies in infancy: The roots of language and musical development. In S. McAdams \& E. Bigand (Eds.), Thinking in sound: Cognitive perspectives on human audition (pp. 278-327). London: Oxford University Press.

TrehUB, S. E., \& UNYK, A. M. (1991). Music prototypes in developmental perspective. Psychomusicology, 10, 31-45.

van de Geer, J. P., Levelt, W. J. M., \& Plomp, R. (1962). The connotation of musical consonance. Acta Psychologica, 20, 308-319.

WARD, W. D. (1970). Musical perception. In J. Tobias (Ed.), Foundations of modern auditory theory (Vol. 1., pp. 407-447). New York: Academic Press.

\section{NOTE}

1. The subscripts denote the octave from which the tone is drawn. $\mathrm{C}_{4}$ is middle $\mathrm{C} ; \mathrm{C}_{1}$, then, is three octaves below middle $\mathrm{C}$.

(Manuscript received September 13, 1993; revision accepted for publication March 7, 1994.) 